\title{
Anesthetic Propofol-Induced Gene Expression Changes in Patients Undergoing Coronary Artery Bypass Graft Surgery Based on Dynamical Differential Coexpression Network Analysis
}

\author{
Da Yu, Li-Jun Huang, and Na-Mi Chen \\ Department of Anesthesiology, Yinzhou Hospital Affiliated to Medical School of Ningbo University, Ningbo, Zhejiang 315000, China
}

Correspondence should be addressed to Da Yu; yudaningbo@sina.com

Received 8 April 2016; Revised 12 June 2016; Accepted 15 June 2016

Academic Editor: Emil Alexov

Copyright (C) 2016 Da Yu et al. This is an open access article distributed under the Creative Commons Attribution License, which permits unrestricted use, distribution, and reproduction in any medium, provided the original work is properly cited.

\begin{abstract}
We aimed to determine the influence of anesthetic propofol on gene expression in patients treated by coronary artery bypass graft (CABG) surgery based on differential coexpression network (DCN) and to further reveal the novel mechanisms of the cardioprotective effects of propofol. Firstly, we constructed the DCN for disease condition based on Pearson correlation coefficient (PCC) and weight value. Secondly, the inference of modules was applied to search modules from DCN with same members but varied connectivity. Furthermore, we measured the statistical significance of the modules for selecting differential modules (DMs). Finally, attract method was used for DMs analysis to select key modules. Based on the $\delta$ value, 11928 edges and 2956 nodes were chosen to construct DCNs. A total of 29 seed genes were selected. Moreover, by quantifying connectivity changes in shared gene modules across different conditions, 8 DMs with higher connectivity dynamics were identified. Then, we extracted key modules using attract method, there were 8 key modules, and the top 3 modules were module 1, 2, and 3. Furthermore, GCG, PPY, and PON1 were initial seed genes of these 3 key modules, respectively. Accordingly, GCG and PON1 might exert important roles in the cardioprotective effects of propofol during CABG.
\end{abstract}

\section{Introduction}

Heart disease is a common, costly, and potentially fatal condition. In developed countries, around $2 \%$ of adults have heart disease and in those over the age of 65, this increases to 6-10\% [1]. At present, with increasing numbers of patients, coronary artery bypass grafting (CABG) has become the most common operation in the world [2]. In order to provide organ protection, the doctors will perform the preoperative anesthesia. Propofol (2,6-diisopropylphenol) is an intravenous sedative agent widely used for anesthesia and sedation [3]. Propofol has antioxidant and antiapoptotic effects [4-6]. In critically ill patients, propofol has been found to be superior to some medicine in both effectiveness and overall cost [7]. At present, it has been verified that propofol can promote the recovery after surgery [8]. Moreover, it has been suggested that propofol attenuates myocardial lipid peroxidation during CABG surgery [9]. Growing evidences have indicated that propofol has effective cardioprotective effects, yet little is known about how propofol is to protect the heart cells from molecular biology aspect during the CABG.

During the past decades, rapid advances in highthroughput technologies have brought unprecedented opportunities for the large-scale analysis of the disease molecular mechanisms. Moreover, network biology has demonstrated to be a powerful tool for analyzing complex molecular networks to identify informative genes which exerts important functions in the development and progression of disease $[10,11]$; for example, coexpression networks are employed in most researches. However, the drawback of coexpression networks may reduce the statistical power to screen pathways which are abnormal in disease conditions. Furthermore, too big network possibly neglects a certain number of important genes and interactions [12], and assessing modules or subnetworks of the intricate network can avoid this 
kind of difficulty [11, 13]. In small module or subnetwork, functions of individual genes and gene-gene interactions may be studied in more detail and precisely [14]. Traditionally, if one gene-gene interaction between a gene pair exhibits highly correlated strength in one state, this interaction will be chosen as an edge of network [15]. Nevertheless, if one gene in the interaction is differently expressed but the other one is not, it may not be regarded as significant interaction for the whole dataset. To a great degree, the difficulty can be solved via constructing differential coexpression network (DCN). Fortunately, Ma et al. [16] offered the module-search method to screen coherently differentially expressed gene modules with common members yet varied connectivity in DCN. Moreover, differential expression network has been created to identify groups of genes that exhibit coherent differential activities between healthy and diseased conditions [17]. In addition, $\mathrm{Li}$ and colleagues used multiple differential expression networks to extract significant genes in rectal cancer [18]. Zhai et al. also used DCN to identify the biomarkers and pathway-related modules in ovarian cancer based on topological centralities [19].

In accordance with the reports in cardioprotection during CABG surgery after propofol treatment, we hypothesized that propofol would elicit genomic responses to cardiac surgery in human hearts. Significantly, gene expression changes were related with clinically crucial cardiovascular biosignatures and to physiologic parameters of cardiac function. The key innovation of this novel method can extract unique and shared modules from differential expression networks. By definition, sets of genes that are differentially expressed in diseased condition but do not show correlated expression pattern will not be identified as a differentially coexpressed gene module [20]. Thus, the dysregulation of differential module (DM) gives more proof to the molecular mechanism of cardioprotective effects of propofol during CABG.

In this paper, in order to explore the dynamics changes in atrial gene expression induced by propofol, we utilized the modules-search algorithm to explore the important modules based on the DCN. Firstly, a dataset from the European Bioinformatics Institute (EMBL-EBI) database (E-GEOD-4386) was downloaded. DCN construction was implemented, followed by the identification of modules via three major steps of seed genes selection, module search by seed expansion and entropy minimization, and refinement of modules. Then, statistical significance of modules was computed to select the differential modules (DMs). Such modules might be helpful to reveal the molecular mechanisms of the cardioprotective effects of propofol during CABG.

\section{Materials and Methods}

2.1. Expression Profile Data Collection. The gene expression profile of E-GEOD-4386 [21] was recruited from the platform of A-AFFY-44-Affymetrix GeneChip Human Genome U133 Plus 2.0 [HG-U133_Plus_2] of the EMBL-EBI database which was recommended by most journals as a repository for data supporting peer-reviewed publications [22]. The 10 atrial samples were collected from patients undergoing
CABG surgery with anesthetic propofol treatment, named as propofol group. Moreover, 10 control samples comprised the same patients prior to CABG surgery, determined as baseline group. Through the mapping between each probe and the corresponding official symbol by getSYMBOL, we got one expression profile data which contained 20389 genes.

We downloaded the human protein-protein interaction networks (PPIN) comprising 787896 interactions from the database Search Tool for the Retrieval of Interacting Genes/Proteins (STRING, http://string-db.org/) which provided a comprehensive, yet quality-controlled collection of protein-protein associations for a large number of organisms [23]. Meanwhile, the expression profile data was mapped to the PPIN and a new PPIN was obtained.

2.2. Construction of DCN. For this disease, the DCN construction was made up of two steps. Firstly, a binary coexpression network was constructed. Then, edge weight was assigned on the basis of differential gene expression between baseline and propofol group.

In this research, we firstly calculated the absolute value of Pearson correlation coefficient (PCC) for these interactions in propofol group. The interactions whose correlations were greater than the predefined threshold $\delta(\delta=0.9)$ were selected to construct the coexpression network.

In addition, edge weights were assigned in the binary coexpression network based on the $P$ value of differential gene expression between two conditions. In this paper, we applied EdgeR [24] to calculate the weight value. The formula was as follows:

$$
w_{i, j}= \begin{cases}\frac{\left(\log P_{i}+\log P_{j}\right)^{1 / 2}}{\left(2 * \max _{l \in V}\left|\log P_{l}\right|\right)^{1 / 2}}, & \text { if } \operatorname{cor}(i, j) \geqslant \delta, \\ 0, & \text { if } \operatorname{cor}(i, j)<\delta,\end{cases}
$$

where $P_{i}$ and $P_{j}$ were $P$ values of differential expression genes $i$ and $j$, respectively. $V$ was the gene set of the coexpression network, and cor $(i, j)$ stood for the absolute value of PCC between genes $i$ and $j$. The genes with higher weights likely participated in a pathway that exhibited differential activities between the two conditions being compared.

2.3. Identification of Modules in DCN. Module is designed for identifying gene modules with common members but varied connectivity across multiple molecular interaction networks [25]. Here we applied the modules algorithm to identify modules. Specific steps were as follows.

Firstly, we performed the seed prioritization for finding the seed genes. Here we ranked the genes in DCN by the topological feature of the gene (degree) and calculated the importance of each gene $i$. The algorithm was defined as

$$
g(i)=\sum_{y \in N_{k}(i)} A_{i j k}^{\prime} g(j),
$$

where $N_{k}(i)$ was the set of neighbors in each network $G_{k}$; $A_{k}^{\prime}$ represented the degree normalized weighted adjacency matrix which was calculated as $A_{k}^{\prime}=D^{-1 / 2} A_{k} D^{1 / 2}$, where 
$D$ was diagonal matrix with $A_{k}$. We calculated a $z$-score for each $g(i)$ and then ranked the genes by averaging the $z$ scores across all networks. Ultimately, the top $1 \%$ genes in the network were selected as the seed genes.

Secondly, module search was implemented according to the seed genes expansion and entropy minimization. For the defined seed gene $v \in V$, we treated it as a module $C$ in the network. After that, we added the gene $u$ which was the neighbor of gene $v$ into the module $C$ and obtained the new module $C^{\prime}$. We defined the entropy change $\Delta H$ as the connectivity of the two modules. The function was defined: $\Delta H\left(C^{\prime}, C\right)=H(C)-H\left(C^{\prime}\right)$.

When $\Delta H\left(C^{\prime}, C\right)>0$, it represented that the addition of gene $u$ improved the connectivity of the module $C$. Similar to the above, we joined all the neighboring gene $u$ with $\Delta H\left(C^{\prime}, C\right)>0$ into the module $C$ till $\Delta H$ was not increased.

Thirdly, the modules were refined. In this step, we removed the modules with the number of nodes $<5$. In addition, to merge overlapping candidate modules, we employed Jaccard index [26] which is the ratio of intersection over union for two sets. In our study, two candidate modules were merged when the Jaccard index was 0.5.

2.4. Analysis of Statistical Significance of Modules. In the current study, we calculated the statistical significance of modules according to the null score distribution of modules generated by random networks. Specific steps were as follows: each randomized network which was made up of edges captured from interactions in DCN was identified, and the number of edges in randomized network was the same as that in DCN. After constructing 100 times randomly for each network, we implemented modules search on the randomized networks to obtain the null distribution of module scores. Next, on the basis of the null distribution, we evaluated the $P$ value of each module as the probability of the module having smaller score by chance. Moreover, we used the BenjaminiHochberg method to correct the $P$ values [27]. Finally, we defined the modules with $P$ value $\leq 0.05$ as the DMs.

2.5. Detection of the Key Modules by Attract Method. Attract is essentially the inverse of more traditional gene expression analysis approaches. In this paper, we used attract method [28] to identify the key modules of propofol on myocardial cell based on the above DMs. On the basis of GSEAANOVA, an ANOVA model [28] was fitted to each gene and the expression of one gene was regulated by a single factor standing for the groups as different levels of this class. According to the ANOVA model, the F-statistic value for gene $m$ is counted:

$$
F^{(m)}=\frac{\mathrm{MSS}_{m}}{\mathrm{RSS}_{m}},
$$

where $\mathrm{MSS}_{m}$ is the mean treatment sum of squares and captures the amount of variation because of the groupspecific effects:

$$
\mathrm{MSS}_{m}=\frac{1}{K-1} \sum_{k=1}^{K} r_{k}\left[y_{\cdot k}^{(m)}-y_{. .}^{(m)}\right]^{2} .
$$

$\mathrm{RSS}_{m}$ represents the residual sum of squares, and it is calculated using the following formula:

$$
\mathrm{RSS}_{m}=\frac{1}{N-K} \sum_{k=1}^{K} \sum_{n=1}^{r_{n}}\left[y_{n k}^{(m)}-y_{. .}^{(m)}\right]^{2}
$$

where $N$ means the total number of samples, and the overall mean is counted:

$$
y_{. .}^{(m)}=\frac{1}{K} \sum_{k=1}^{K}\left(\frac{1}{r_{k}} \sum_{n=1}^{r_{k}} y_{n k}^{(m)}\right) .
$$

Here, large value of the $F$-statistic suggested a strong group-specific expression change. A small $F$-statistic was in a similar way. Next, $t$-test was performed for the $\log _{2}$ transformed $F$-statistics values from above the modules. Moreover, Benjamini-Hochberg-based method was utilized to adjust the $P$ values [27]. In our analysis, the modules with adjusted $P$ values $\leq 0.05$ were considered as key modules.

2.6. Pathway Enrichment Analysis. The occurrence of diseases is often induced by the dysregulation of pathways involved in the biological process. Because of this, we focused on the pathways enrichment analysis for key module genes to narrow down our analysis. Kyoto Encyclopedia of Genes and Genomes (KEGG) database provides a reference knowledge for understanding biological processes via pathway aligning, which is to align genes to reference pathways to deduce the behaviors of cell [29]. In our study, all reference pathways were downloaded from KEGG database. Then, genes in key modules were mapped to the reference pathways to identify the abnormal pathways. Significant pathways of the genes in key modules between two groups were identified when the FDR was set as 0.01 and the number of genes in pathways was not less than 10 .

\section{Results}

3.1. Construction of DCN. In this paper, a total of 787896 humans PPIs (16730 genes) were downloaded from STRING database. After the genes in microarray data were aligned to the ensemble PPIN, a new PPIN including 15171 genes and 728200 interactions was obtained. In order to construct coexpression network, edges were extracted according to the absolute value of the PCC of the expression profiles of two genes. Based on the absolute value $>0.9$, we selected 11928 interactions and 2956 genes to construct the DCN.

3.2. Identification of Modules in DCN. Based on $z$-score of each gene, we selected a total of 29 seed genes in DCN. The result was shown in Table 1. Among these 29 seed genes, there were 4 genes with $z$-score value $>110$, for example, CSN1S1 $(z$-score $=139.75), \operatorname{ALB}(z$-score $=130.93), \operatorname{ALPP}(z$ score $=120.37)$, and CDH1 $(z$-score $=113.58)$. Subsequently, taking these 29 seed genes as start, we implemented module identification based on the entropy decrease $\Delta H\left(C^{\prime}, C\right)$. Moreover, according to the entropy change, we showed that gene modules with higher connectivity dynamics were under 
TABLE 1: The 29 seed genes identified in the differential coexpression network (DCN) and the distribution of their $z$-scores.

\begin{tabular}{lc}
\hline Seed genes & $z$-score \\
\hline CSN1S1 & 139.75 \\
ALB & 130.93 \\
ALPP & 120.37 \\
CDH1 & 113.58 \\
IL2 & 107.69 \\
ERBB2 & 99.76 \\
IL4 & 94.42 \\
CYP3A43 & 94.21 \\
MPO & 90.49 \\
ATP4A & 86.75 \\
PPY & 82.40 \\
GFAP & 77.59 \\
SLC6A7 & 76.27 \\
CYP3A5 & 75.42 \\
GRP & 75.17 \\
GCG & 73.66 \\
IL1A & 72.32 \\
DNAH8 & 71.73 \\
PON1 & 71.38 \\
NANOG & 70.21 \\
PTH & 67.75 \\
KNG1 & 66.82 \\
SERPINC1 & 66.70 \\
PRL & 66.12 \\
REN & 66.08 \\
TPH1 & 63.74 \\
GJB6 & 62.07 \\
MYB & 61.89 \\
SULT2A1 & 61.44 \\
\hline
\end{tabular}

$\Delta H\left(C^{\prime}, C\right)>0$. In this research, we obtained 8 modules after removing the modules with the number of nodes $<5$ as well as merging into one module if the Jaccard index $\geq 0.5$ between the two modules.

3.3. Analysis of Statistical Significance of Modules. After constructing 100 times randomly for each network, we searched 5845 modules in all. Through the calculation and correction of the $P$ value of all modules, at a $P$ value threshold of 0.05 , we screened several modules as DMs. What was more, we noticed that all the above 8 modules were DMs owing to their $P$ value $<0.05$ after the statistical analysis. The result was shown in Table 2.

3.4. Detection of the Key Modules by Attract Method. In this part, we evaluated the adjusted $P$ values for these $8 \mathrm{DMs}$ utilizing the attract way. We found that these $8 \mathrm{DMs}$ were key modules. In order to further verify the connectivity dynamics of two genes in key modules, we focused on the weight distribution of edges (Table 3). Significantly, we found that a majority of interactions distributed in the range of 0.10.2 , which indicated that genes were connected with each
TABLE 2: The 8 differential modules (DMs) with adjusted $P$ values < 0.5 and the initial seed genes in the DMs.

\begin{tabular}{lcc}
\hline Modules & Adjusted $P$ values & Initial seed gene \\
\hline Module 1 & $1.12 E-11$ & GCG \\
Module 2 & $3.43 E-09$ & PPY \\
Module 3 & $6.21 E-09$ & PON1 \\
Module 4 & $1.32 E-07$ & IL1A \\
Module 5 & $2.59 E-07$ & GJB6 \\
Module 6 & $7.97 E-06$ & GRP \\
Module 7 & $7.97 E-06$ & TPH1 \\
Module 8 & $2.23 E-05$ & SULT2A1 \\
\hline
\end{tabular}

other closely, and these key modules had good connectivity properties in propofol group. Here, the top 3 modules were module 1 (adjusted $P$ value $=1.12 E-11)$, module 2 (adjusted $P$ value $=3.43 E-09)$, and module 3 (adjusted $P$ value $=$ $6.21 E-09)$. Meanwhile, among the 3 key modules, GCG, PPY, and PON1 were initial seed genes, respectively. Here we showed the 3 key modules in Figure 1.

3.5. Pathway Enrichment Analysis. To further research the dysregulated biological functions in disease condition, pathway analysis was conducted based on the genes in key modules. Based on FDR $<0.01$ and the number of genes $\geq 10$, genes in key modules $1,2,3,5,6$, and 7 were simultaneously enriched in the pathway of neuroactive ligand-receptor interaction. The gene count and the FDR were shown in Table 4.

\section{Discussion}

It has been reported that anesthetic modulates gene expression [30] and provided organ protection [31]. Propofol is a general anesthetic widely used for the induction and maintenance of anesthesia during cardiac surgery and for postoperative sedation. Furthermore, several researches have previously published that propofol conferred protection against damage to the myocardium for patients undergoing CABG [32-34]. However, the related protection mechanisms from the molecular dynamics level were rarely involved. This study aimed at identifying myocardial transcriptional phenotypes to predict dynamics variation of the biomarkers and function in myocardial cell taking propofol anesthesia undergoing CABG surgery. In our research, the key innovation is the ability to identify several important modules from DCN, each of which representing a different perturbation condition. Here we noticed that genes in 8 DMs had more connections and located in more central positions in the network. Particularly, the 3 key modules had higher correlation with the disease phenotype. What is more, among the 3 modules, GCG, PPY, and PON1 were initial seed genes, respectively. These data underlined the importance of these genes and their corresponding modules for the research of dynamic variation in myocardial cell induced by propofol.

GCG (glucagon) is a protein coding gene, which plays a key role in glucose metabolism and homeostasis. It has been reported that the secretion of glucagon-like peptide- 1 and 
TABLE 3: The distribution of weight values of 8 key modules.

\begin{tabular}{lccc}
\hline Modules & Number of interactions & Number of interactions & Number of interactions \\
& $0-0.1$ & $0.1-0.2$ & $0.2-0.3$ \\
\hline Module 1 & 0 & 865 & 1 \\
Module 2 & 1 & 764 & 1 \\
Module 3 & 1 & 645 & 3 \\
Module 4 & 1 & 742 & 0 \\
Module 5 & 1 & 531 & 3 \\
Module 6 & 0 & 731 & 1 \\
Module 7 & 4 & 441 & 1 \\
Module 8 & 3 & 693 & 1 \\
\hline
\end{tabular}

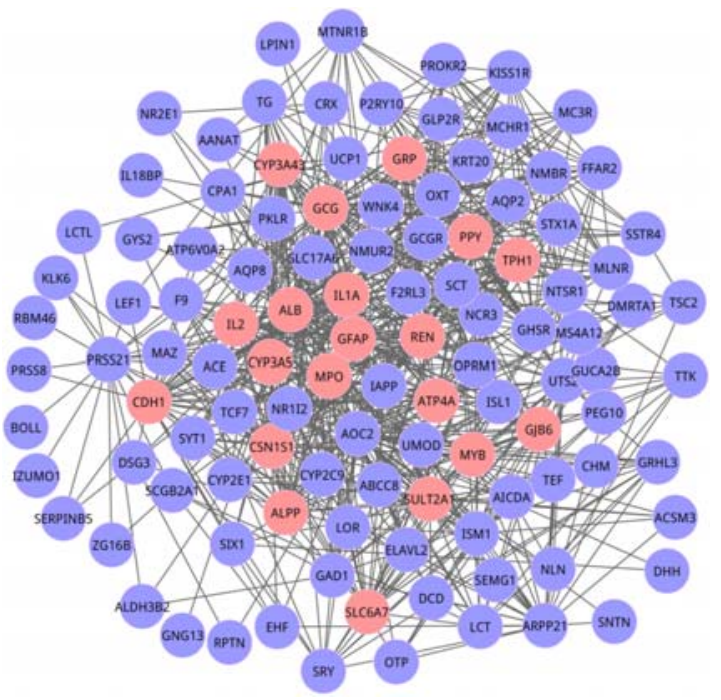

(a)

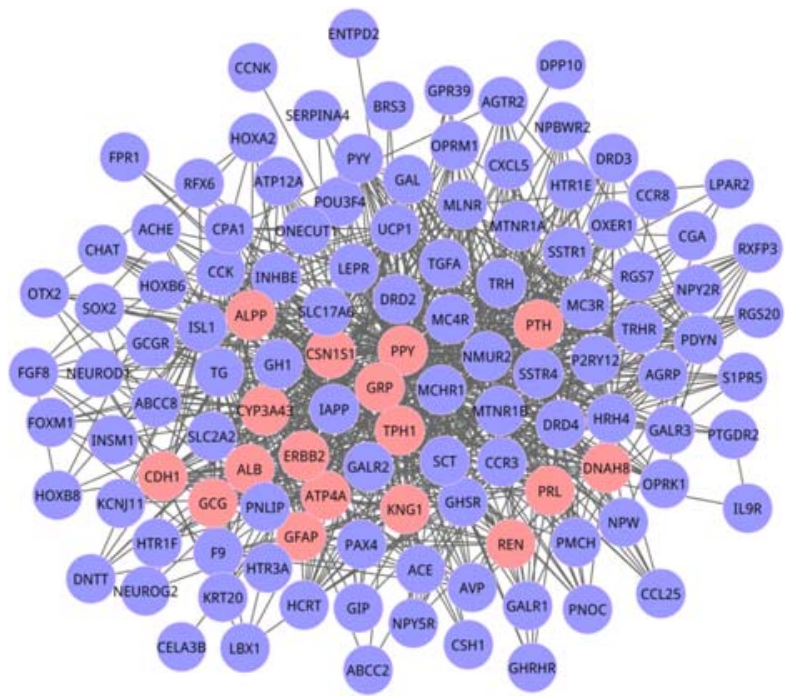

(b)

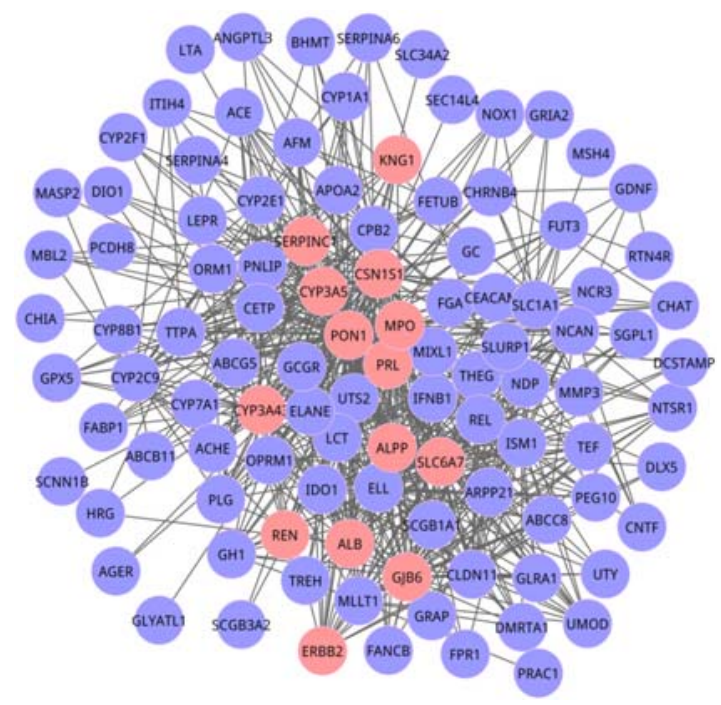

(c)

FIGURE 1: The 3 key modules identified in the differential coexpression network (DCN) of baseline and propofol group based on the adjusted $P$ values by attract method. (a), (b), and (c) represented module 1, module 2 , and module 3 , respectively. Nodes were genes, and edges were the interactions among two genes. There were 119 nodes and 866 edges in module 1; 112 nodes and 766 edges were contained in module 2; module 3 consisted of 105 nodes and 649 edges. The pink nodes were seed genes. 
TABLE 4: Kyoto Encyclopedia of Genes and Genomes (KEGG) pathway enrichment analysis of genes in key modules based on false discovery rate $(\mathrm{FDR})<0.01$ and gene count $\geq 10$.

\begin{tabular}{|c|c|c|c|}
\hline Modules & KEGG pathways & Gene count & FDR \\
\hline Module 1 & Neuroactive ligand-receptor interaction & 15 & $8.32 E-06$ \\
\hline Module 2 & Neuroactive ligand-receptor interaction & 37 & $7.10 E-26$ \\
\hline Module 3 & Neuroactive ligand-receptor interaction & 10 & $1.63 E-03$ \\
\hline \multirow{4}{*}{ Module 4} & Cytokine-cytokine receptor interaction & 29 & $6.91 E-16$ \\
\hline & JAK-STAT signaling pathway & 20 & $1.73 E-11$ \\
\hline & Toll-like receptor signaling pathway & 11 & $4.32 E-05$ \\
\hline & Natural killer cell mediated cytotoxicity & 12 & $6.04 E-05$ \\
\hline Module 5 & Neuroactive ligand-receptor interaction & 10 & $3.76 E-03$ \\
\hline Module 6 & Neuroactive ligand-receptor interaction & 33 & $4.62 E-25$ \\
\hline Module 7 & Neuroactive ligand-receptor interaction & 16 & $2.11 E-07$ \\
\hline Module 8 & Steroid hormone biosynthesis & 11 & $4.36 E-09$ \\
\hline
\end{tabular}

glucose-dependent insulinotropic polypeptide is under influence of the digestion and absorption of nutrients in the small intestine and that pancreatic enzyme substitution increased insulin secretion [35]. Concurrently, Jeppesen et al. [36] have demonstrated that glucagon-like peptide- 2 treatment reduces fecal weight and enabled short bowel syndrome patients to maintain their intestinal fluid. Subsequent researches have shown that glucagon-like peptide-1 infusion improved regional and global left ventricular function in patients with acute myocardial infarction and severe systolic dysfunction after successful primary angioplasty [37]. Significantly, Kitamura et al. [38] have indicated that propofol contributes to the stable glucose metabolism during surgery. In light of these, we infer that propofol might play important roles in cardioprotective effects, at least in part, through regulating GCG expression.

Serum paraoxonase 1 (PON1) also known as A esterase, homocysteine thiolactonase, or serum aryldialkylphosphatase 1 is an enzyme that in humans is encoded by the PON1 gene [39]. Decades of research have indicated that PON1 protected humans from the acute and chronic harmful effects of a variety of disease. For example, it has been verified that PON1 is also a major antiatherosclerotic component of high-density lipoprotein [40, 41]. Li et al. [42] suggested that subjects of low PON1 activity may be more susceptible to arsenicrelated cardiovascular disease. Moreover, significant decrease of PON1 activity confirmed the high risk of cardiovascular diseases in smokers [43]. What is more, the evaluation of PON1 activity demonstrated its decrease was a risk factor associated with increased coronary heart disease susceptibility $[44,45]$. In this research, compared with the control cases without propofol after CABG surgery, we investigated the disease group received the intravenous anesthetic propofol after undergoing $\mathrm{CABG}$ surgery and showed the crucial gene PON1 in 3-DM. Hence, it provided conclusive evidence on the effects of propofol on heart disease by altering the activity of PON1.

Significantly, in the current study, pathway enrichment results indicated that genes in key modules $1,2,3,5,6$, and 7 were simultaneously enriched in the pathway of neuroactive ligand-receptor interaction. As documented, the pathway of neuroactive ligand-receptor interaction has been demonstrated to be associated with the development and progress of cardiovascular diseases [46]. The key proteins in this pathway, such as angiotensin, adrenergic, and calcitonin receptor-like neurotensin receptors, have also been indicated to be closely related to cardiac function [47, 48]. Recently, growing evidence has suggested that propofol exerts direct inhibitory effects on adrenergic receptor signal transduction in cardiomyocytes [49]. Accordingly, we infer that these key modules might be beneficial to reveal the cardioprotective effects of propofol during CABG.

\section{Conclusion}

Taken together, these key modules and their seed genes might exert important roles in the cardioprotective effects of propofol. However, the identified modules and their initial seed genes in this study were not confirmed in animal models. Further study in animal models may be required in the later work.

\section{Competing Interests}

The authors state they have no competing interests.

\section{References}

[1] K. Dickstein, A. Cohen-Solal, G. Filippatos et al., "ESC Guidelines for the diagnosis and treatment of acute and chronic heart failure 2008," European Journal of Heart Failure, vol. 10, no. 10, pp. 933-989, 2008.

[2] A. Rezaianzadeh, B. Maghsoudi, H. Tabatabaee et al., "Factors associated with extubation time in coronary artery bypass grafting patients," Peer Journal, vol. 3, article el414, 2015.

[3] C. L. Xie, Y. B. Pan, L. Q. Hu, and Y. N. Qian, "Propofol attenuates hydrogenperoxide-induced apoptosis in human umbilical vein endothelial cells via multiple signaling pathways," Korean Journal of Anesthesiology, vol. 68, no. 5, pp. 488-495, 2015.

[4] J. Chen, Y. Gu, Z. Shao, J. Luo, and Z. Tan, "Propofol protects against hydrogen peroxide-induced oxidative stress and cell dysfunction in human umbilical vein endothelial cells," Molecular and Cellular Biochemistry, vol. 339, no. 1-2, pp. 43-54, 2010. 
[5] D. M. Ansley, J. Sun, W. A. Visser et al., "High dose propofol enhances red cell antioxidant capacity during CPB in humans," Canadian Journal of Anaesthesia, vol. 46, no. 7, pp. 641-648, 1999.

[6] B. Wang, J. Shravah, H. Luo, K. Raedschelders, D. D. Y. Chen, and D. M. Ansley, "Propofol protects against hydrogen peroxide-induced injury in cardiac $\mathrm{H} 9 \mathrm{c} 2$ cells via Akt activation and Bcl-2 up-regulation," Biochemical and Biophysical Research Communications, vol. 389, no. 1, pp. 105-111, 2009.

[7] C. E. Cox, S. D. Reed, J. A. Govert et al., "An economic evaluation of propofol and lorazepam for critically ill patients undergoing mechanical ventilation," Critical Care Medicine, vol. 36, no. 3, pp. 706-714, 2008.

[8] J. G. Klamt, L. V. Garcia, R. M. Stocche, and M. P. Dos Reis, "Continuous epidural anesthesia with $0.2 \%$ Ropivacaine associated to general anesthesia for upper abdominal surgery in children," Revista Brasileira de Anestesiologia, vol. 53, no. 2, pp. 160-168, 2003.

[9] M. M. Sayin, O. Özatamer, R. Taöz, K. Kilinç, and N. Ünal, "Propofol attenuates myocardial lipid peroxidation during coronary artery bypass grafting surgery," British Journal of Anaesthesia, vol. 89, no. 2, pp. 242-246, 2002.

[10] D. He, Z.-P. Liu, M. Honda, S. Kaneko, and L. Chen, "Coexpression network analysis in chronic hepatitis B and $\mathrm{C}$ hepatic lesions reveals distinct patterns of disease progression to hepatocellular carcinoma," Journal of Molecular Cell Biology, vol. 4, no. 3, pp. 140-152, 2012.

[11] Z.-P. Liu, Y. Wang, X.-S. Zhang, and L. Chen, "Network-based analysis of complex diseases," IET Systems Biology, vol. 6, no. 1, pp. 22-33, 2012.

[12] R. K. Nibbe, S. A. Chowdhury, M. Koyutürk, R. Ewing, and M. R. Chance, "Protein-protein interaction networks and subnetworks in the biology of disease," Wiley Interdisciplinary Reviews: Systems Biology and Medicine, vol. 3, no. 3, pp. 357-367, 2011.

[13] Y. Wu, R. Jing, L. Jiang et al., "Combination use of proteinprotein interaction network topological features improves the predictive scores of deleterious non-synonymous singlenucleotide polymorphisms," Amino Acids, vol. 46, no. 8, pp. 2025-2035, 2014.

[14] V. Emilsson, G. Thorleifsson, B. Zhang et al., "Genetics of gene expression and its effect on disease," Nature, vol. 452, no. 7186, pp. 423-428, 2008.

[15] L. Chen, R.-S. Wang, and X.-S. Zhang, “Transcription regulation: networks and models," in Biomolecular Networks, pp. 2345, John Wiley \& Sons, New York, NY, USA, 2009.

[16] X. Ma, L. Gao, G. Karamanlidis et al., "Revealing pathway dynamics in heart diseases by analyzing multiple differential networks," PLoS Computational Biology, vol. 11, no. 6, Article ID e1004332, 2015.

[17] S.-Y. Sun, Z.-P. Liu, T. Zeng, Y. Wang, and L. Chen, "Spatiotemporal analysis of type 2 diabetes mellitus based on differential expression networks," Scientific Reports, vol. 3, article 2268, 2013.

[18] R.-H. Li, A. Zhang, S. Li et al., "Multiple differential expression networks identify key genes in rectal cancer," Cancer Biomarkers, vol. 16, no. 3, pp. 435-444, 2016.

[19] D. K. Zhai, B. Liu, X. F. Bai, and J. A. Wen, "Identification of biomarkers and pathway-related modules involved in ovarian cancer based on topological centralities," Journal of B.U.ON., vol. 21, no. 1, pp. 208-220, 2016.
[20] E. Segal, M. Shapira, A. Regev et al., "Module networks: identifying regulatory modules and their condition-specific regulators from gene expression data," Nature Genetics, vol. 34, no. 2, pp. 166-176, 2003.

[21] E. Lucchinetti, C. Hofer, L. Bestmann et al., "Gene regulatory control of myocardial energy metabolism predicts postoperative cardiac function in patients undergoing off-pump coronary artery bypass graft surgery: inhalational versus intravenous anesthetics," Anesthesiology, vol. 106, no. 3, pp. 444-457, 2007.

[22] N. Kolesnikov, E. Hastings, M. Keays et al., "ArrayExpress update-simplifying data submissions," Nucleic Acids Research, vol. 43, no. 1, pp. D1113-D1116, 2015.

[23] C. Von Mering, L. J. Jensen, B. Snel et al., "STRING: known and predicted protein-protein associations, integrated and transferred across organisms," Nucleic Acids Research, vol. 33, supplement 1, pp. D433-D437, 2005.

[24] M. D. Robinson, D. J. McCarthy, and G. K. Smyth, "edgeR: a bioconductor package for differential expression analysis of digital gene expression data," Bioinformatics, vol. 26, no. 1, pp. 139-140, 2010.

[25] X. Ma, L. Gao, and K. Tan, "Modeling disease progression using dynamics of pathway connectivity," Bioinformatics, vol. 30, no. 16, pp. 2343-2350, 2014.

[26] M. Bouchard, A.-L. Jousselme, and P.-E. Doré, "A proof for the positive definiteness of the Jaccard index matrix," International Journal of Approximate Reasoning, vol. 54, no. 5, pp. 615-626, 2013.

[27] Y. Benjamini and Y. Hochberg, "Controlling the false discovery rate: a practical and powerful approach to multiple testing," Journal of the Royal Statistical Society, Series B: Methodological, vol. 57, no. 1, pp. 289-300, 1995.

[28] J. C. Mar, N. A. Matigian, J. Quackenbush, and C. A. Wells, "Attract: a method for identifying core pathways that define cellular phenotypes," PLoS ONE, vol. 6, no. 10, Article ID e25445, 2011.

[29] M. Kanehisa, M. Araki, S. Goto et al., "KEGG for linking genomes to life and the environment," Nucleic Acids Research, vol. 36, no. 1, pp. D480-D484, 2008.

[30] A. Metaxas, H. L. Keyworth, J. H. Yoo, Y. Chen, I. Kitchen, and A. Bailey, "The stereotypy-inducing and OCD-like effects of chronic 'binge' cocaine are modulated by distinct subtypes of nicotinic acetylcholine receptors," British Journal of Pharmacology, vol. 167, no. 2, pp. 450-464, 2012.

[31] L. Ruggeri, G. Landoni, F. Guarracino, S. Scolletta, E. Bignami, and A. Zangrillo, "Remifentanil in critically ill cardiac patients," Annals of Cardiac Anaesthesia, vol. 14, no. 1, pp. 6-12, 2011.

[32] A. Samir, N. Gandreti, M. Madhere, A. Khan, M. Brown, and V. Loomba, "Anti-inflammatory effects of propofol during cardiopulmonary bypass: a pilot study," Annals of Cardiac Anaesthesia, vol. 18, no. 4, pp. 495-501, 2015.

[33] N. Aghdaii, M. Ziyaeifard, S. Z. Faritus, and R. Azarfarin, "Hemodynamic responses to two different anesthesia regimens in compromised left ventricular function patients undergoing coronary artery bypass graft surgery: etomidate-midazolam versus propofol-ketamine," Anesthesiology and Pain Medicine, vol. 5, no. 3, Article ID e27966, 2015.

[34] Z. E. Plummer, S. Baos, C. A. Rogers et al., "The effects of propofol cardioplegia on blood and myocardial biomarkers of stress and injury in patients with isolated coronary artery bypass grafting or aortic valve replacement using cardiopulmonary bypass: protocol for a single-center randomized controlled trial," JMIR Research Protocols, vol. 3, no. 3, article e35, 2014. 
[35] F. K. Knop, T. Vilsbøll, S. Larsen et al., "Increased postprandial responses of GLP-1 and GIP in patients with chronic pancreatitis and steatorrhea following pancreatic enzyme substitution," American Journal of Physiology-Endocrinology and Metabolism, vol. 292, no. 1, pp. E324-E330, 2007.

[36] P. B. Jeppesen, P. Lund, I. B. Gottschalck et al., "Short bowel patients treated for two years with glucagon-like peptide 2: effects on intestinal morphology and absorption, renal function, bone and body composition, and muscle function," Gastroenterology Research and Practice, vol. 2009, Article ID 616054, 12 pages, 2009.

[37] L. A. Nikolaidis, S. Mankad, G. G. Sokos et al., "Effects of glucagon-like peptide-1 in patients with acute myocardial infarction and left ventricular dysfunction after successful reperfusion," Circulation, vol. 109, no. 8, pp. 962-965, 2004.

[38] T. Kitamura, M. Ogawa, G. Kawamura, K. Sato, and Y. Yamada, "The effects of sevoflurane and propofol on glucose metabolism under aerobic conditions in fed rats," Anesthesia and Analgesia, vol. 109, no. 5, pp. 1479-1485, 2009.

[39] S. L. Primo-Parmo, R. C. Sorenson, J. Teiber, and B. N. La Du, "The human serum paraoxonase/arylesterase gene (PON1) is one member of a multigene family," Genomics, vol. 33, no. 3, pp. 498-507, 1996.

[40] G. S. Getz and C. A. Reardon, "Paraoxonase, a cardioprotective enzyme: continuing issues," Current Opinion in Lipidology, vol. 15, no. 3, pp. 261-267, 2004.

[41] M. Mackness and B. Mackness, "Paraoxonase 1 and atherosclerosis: is the gene or the protein more important?" Free Radical Biology and Medicine, vol. 37, no. 9, pp. 1317-1323, 2004.

[42] W.-F. Li, C.-W. Sun, T.-J. Cheng, K.-H. Chang, C.-J. Chen, and S.-L. Wang, "Risk of carotid atherosclerosis is associated with low serum paraoxonase (PON1) activity among arsenic exposed residents in Southwestern Taiwan," Toxicology and Applied Pharmacology, vol. 236, no. 2, pp. 246-253, 2009.

[43] D. H. Mouhamed, A. Ezzaher, M. Araoud et al., "Paraoxonase 1 (PON1) activity and lipid parameters in Tunisian smokers," Annales de Biologie Clinique, vol. 68, no. 2, pp. 143-147, 2009.

[44] M. Wang, X. Lang, S. Cui et al., "Quantitative assessment of the influence of paraoxonase 1 activity and coronary heart disease risk," DNA and Cell Biology, vol. 31, no. 6, pp. 975-982, 2012.

[45] Y. Zhao, Y. Ma, Y. Fang et al., "Association between PON1 activity and coronary heart disease risk: a meta-analysis based on 43 studies," Molecular Genetics and Metabolism, vol. 105, no. 1, pp. 141-148, 2012.

[46] J. Wei, P. Ramanathan, I. C. Martin, C. Moran, R. M. Taylor, and P. Williamson, "Identification of gene sets and pathways associated with lactation performance in mice," Physiological Genomics, vol. 45, no. 5, pp. 171-181, 2013.

[47] J. P. Vázquez-Medina, I. Popovich, M. A. Thorwald et al., "Angiotensin receptor-mediated oxidative stress is associated with impaired cardiac redox signaling and mitochondrial function in insulin-resistant rats," American Journal of PhysiologyHeart and Circulatory Physiology, vol. 305, no. 4, pp. H599H607, 2013.

[48] Y. Wang, X. Yu, F. Wang et al., "Yohimbine promotes cardiac NE release and prevents LPS-induced cardiac dysfunction via blockade of presynaptic $\alpha 2 \mathrm{~A}$-adrenergic receptor," PLoS ONE, vol. 8, no. 5, Article ID e63622, 2013.

[49] W. Zhou, H. J. Fontenot, S.-N. Wang, and R. H. Kennedy, "Propofol-induced alterations in myocardial $\beta$-adrenoceptor binding and responsiveness," Anesthesia \& Analgesia, vol. 89, no. 3, pp. 604-608, 1999. 


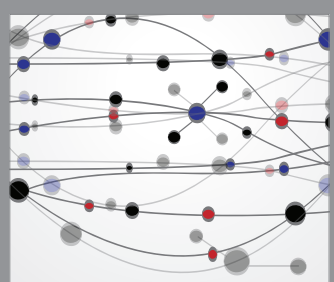

The Scientific World Journal
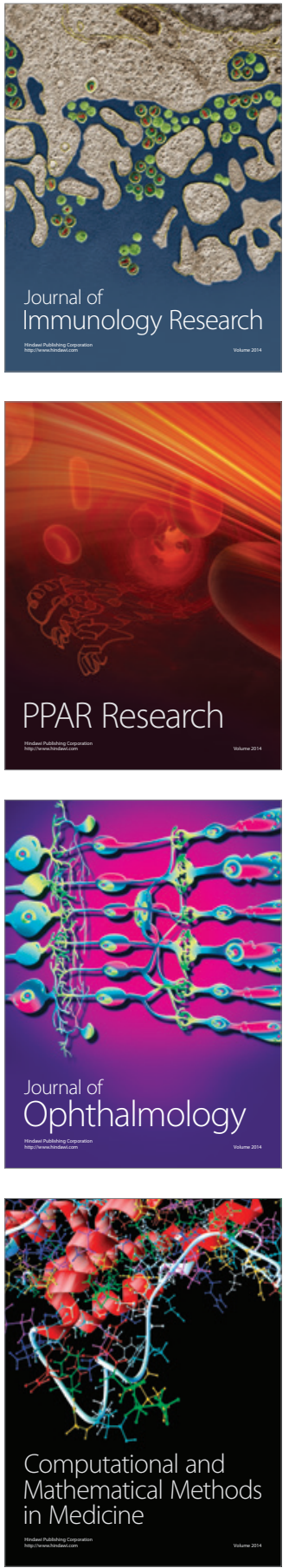

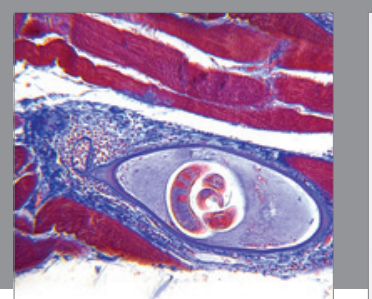

Gastroenterology Research and Practice

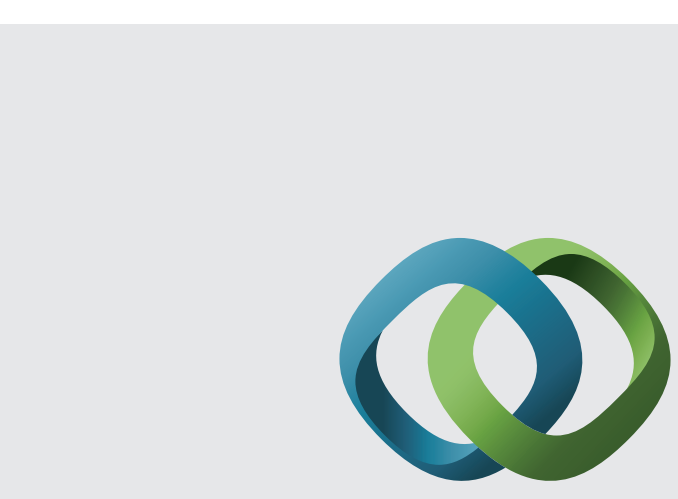

\section{Hindawi}

Submit your manuscripts at

http://www.hindawi.com
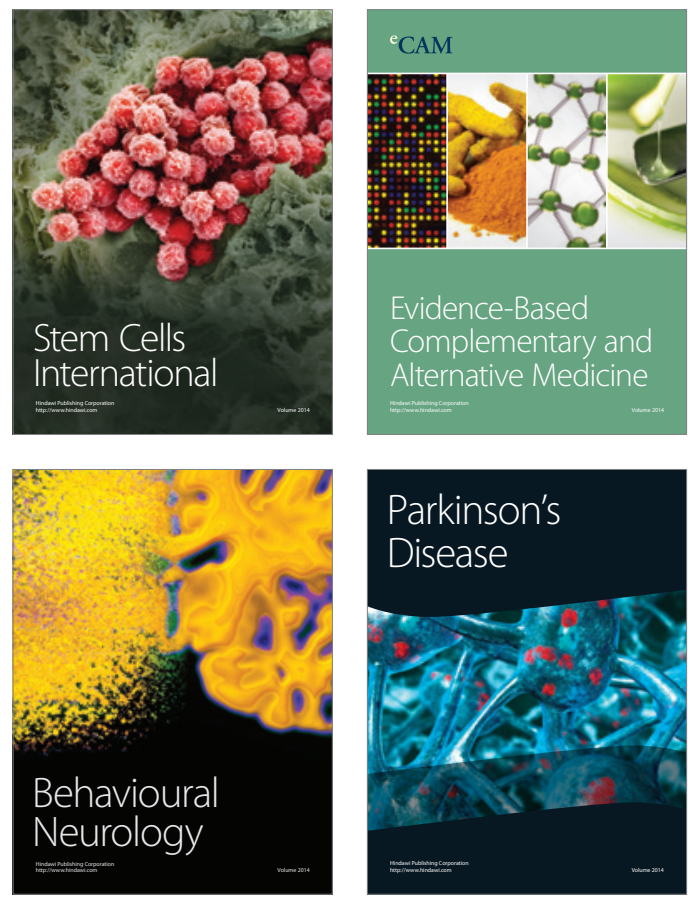
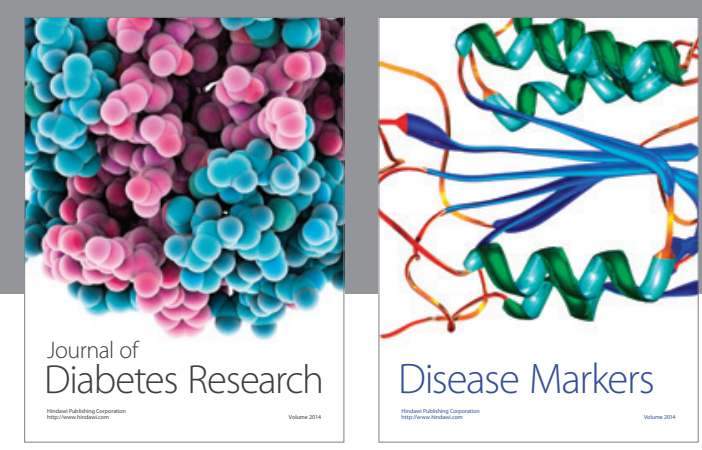

Disease Markers
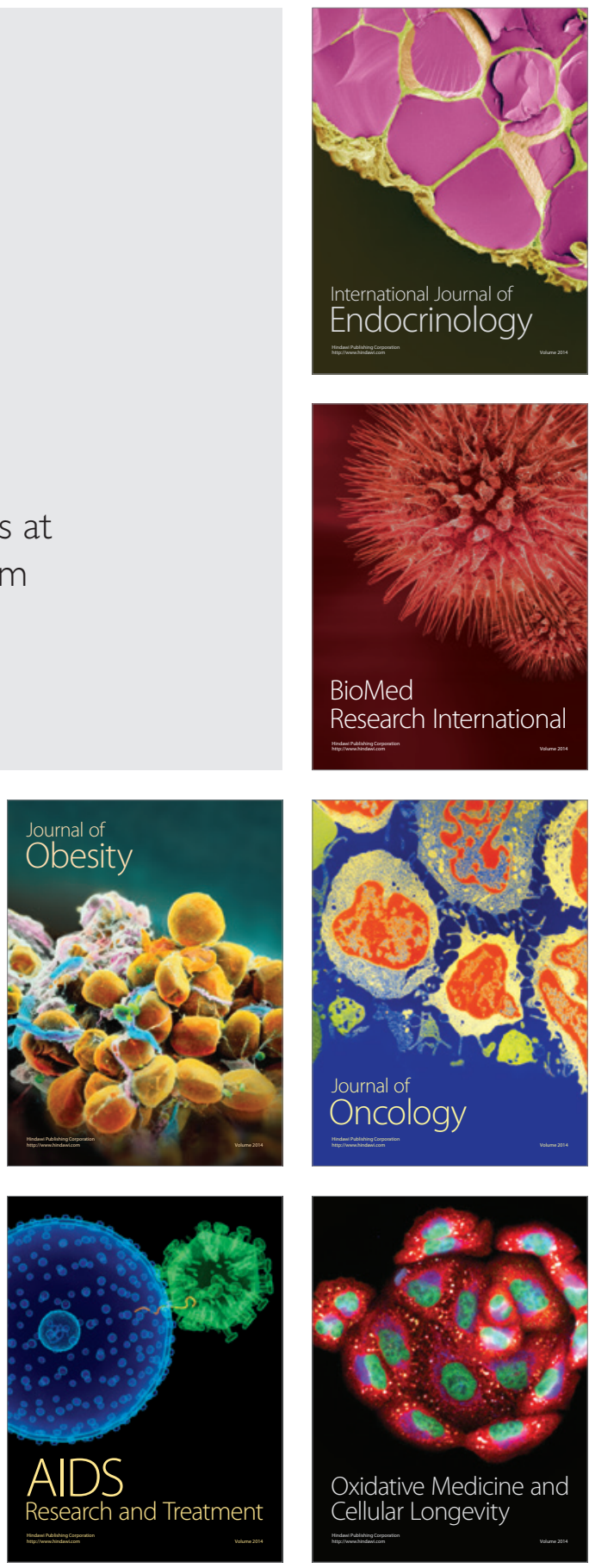